\title{
Parametric Analysis of CO2 Laser for Quality of Weldox- 700 Material
}

\author{
Vadhel Ajay Jethabhai \\ Department of Mechanical Engineering \\ Indus Institute of Technology and Engineering \\ Ahmedabad, Gujarat, India
}

\author{
Jignesh Patel \\ Department of Mechanical Engineering \\ Indus Institute of Technology and Engineering \\ Ahmedabad, Gujarat, India
}

\begin{abstract}
Laser Cutting of weldox-700 materials is popular processes in manufacturing industries This research paper for primary study for evaluate the effect of the processing parameters (Laser Power, gas pressure, pulse frequency and Cutting speed) under the quality of the cut for weldox-700 materials. Then the experiment was done by $6 \mathrm{~mm}$ thick weldox-700 material plates. In this experiment focused on establish relation of different parameters on Cutting quality, which is decided by the Surface Roughness and Kerf Width.
\end{abstract}

Keywords: Weldox-700, Cutting Speed, Cutting Quality, Laser Power, Taguchi method, Surface Roughness, Kerf Width

\section{INTRODUCTION :}

Laser Cutting machine definition describes itself "Laser machine is a device which is used to generate \& amplifies light. Laser stands for Light Amplification by Stimulated Emission of Radiation. Laser machine is an electrical-optical device that produces coherent radiation. Simply put, a Laser is a device that creates and amplifies a narrow, intense beam of coherent light" [1].In the industries the high accuracy of Cutting, higher production rate, good Cutting quality this all thing proves that $\mathrm{co} 2$ Laser machine is good choice for industrial application. There are many parameters are affected like Laser Power, Cutting speed, gas pressure, pulse frequency, thickness of material plates, focus length then after measure Surface Roughness and Kerf Widths References[2] give detailed descriptions of the Laser machine and the mechanism of Laser Cutting of materials an Fig. 1shows Schematic illustration for the process of co2 Laser Cutting of metals. In this research paper Cutting of hardest material Weldox-700 is selected which is used in earth moving machine parts. Weldox-700 has hardness of $700 \mathrm{HBW}$; it has also very good weld ability and good hardness. So it is used in dumper bodies, mining equipment, water tanks, concreter crusher and over head cranes [3].

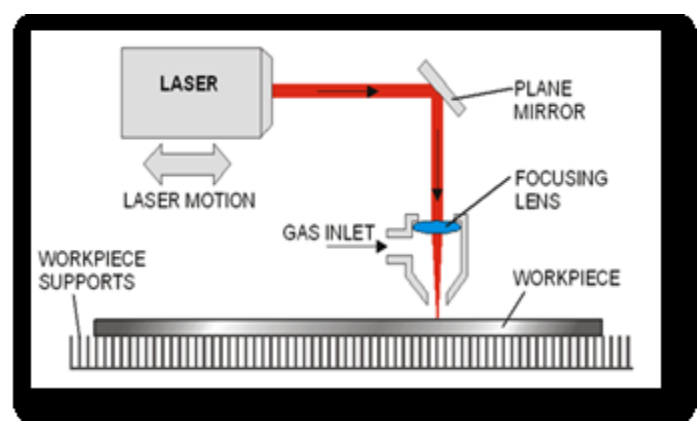

Figure 1: Schematic illustration for the process of CO2 Laser Cutting of metals

\section{EXPERIMENTAL PROCEDURE AND OPERATION PARAMETERS :}

\subsection{Material :}

The base material used in this study was weldox-700 sheet 6 $\mathrm{mm}$ thick, whose chemical composition listed in Table 1. This weldox-700[3].

\begin{tabular}{|c|c|c|c|c|c|c|}
\hline $\mathrm{C}$ & $\mathrm{Si}$ & $\mathrm{Mn}$ & $\mathrm{P}$ & $\mathrm{S}$ & $\mathrm{Mo}$ & $\mathrm{Cu}$ \\
\hline $\mathbf{0 . 2 0}$ & $\mathbf{0 . 6 0}$ & $\mathbf{1 . 6 0}$ & $\mathbf{0 . 0 2 0}$ & $\mathbf{0 . 0 1 0}$ & $\mathbf{0 . 7 0}$ & $\mathbf{0 . 3 0}$ \\
\hline
\end{tabular}

Table 1: chemical composition of weldox-700

\subsection{Taguchi method based experiment :}

These experiments were performed with a $3.2 \mathrm{~kW}$ $\mathrm{CO} 2$, To prevent the instability and damage caused by back reflections, the cavity is isolated by using a beam bender mirror with a multilayer coating that absorbs the back reflected Laser beam. The Laser beam was focused using a $127 \mathrm{~mm}$ focal length lens except for the tests conducted to detect the influence of this parameter. In CW mode, when the Laser source delivers a constant Power, the experiments were performed varying one factor at a time. The ranges of Cutting parameter are summarized in Table 2. A commercial Cutting head incorporating a conical converging coaxial nozzle with a $1.5 \mathrm{~mm}$ exit diameter was employed to supply the assist gas in a coaxial manner with the Laser beam. In the tests conducted to reveal the influence of the nozzle exit on the quality of the cuts, nozzles with an exit diameter of $1.5 \mathrm{~mm}$ were also used. The distance from the lower part of the nozzle to the plate was fixed at $1.5 \mathrm{~mm}$ except for the tests conducted to reveal the influence of this parameter. Compressed air, nitrogen and oxygen at various pressures were used as assist gases. Based on 
experience for similar materials, oxygen and nitrogen are the most recommended gases for the experimentation [4].

Most of the tests were performed using argon as assist gas except from the test performed to investigate the influence of the gas nature. In order to compare the results, the experiments were performed on unidirectional straight line Cutting. On the other hand, the experiments conducted in pulsed mode were performed by means of one-factor-at-a-time experiments in a first stage. The ranges of Cutting parameters are summarized in Table 2 Furthermore; a full factorial design (FFD) approach was also performed to efficiently screen out the key variables significantly affecting on the response variables.

\begin{tabular}{|c|c|c|c|c|c|}
\hline Symbol & Factor & Unit & $\begin{array}{c}\text { Level } \\
1\end{array}$ & $\begin{array}{c}\text { Level } \\
\mathbf{2}\end{array}$ & $\begin{array}{c}\text { Level } \\
\mathbf{3}\end{array}$ \\
\hline A & Power & Watt & $\mathbf{1 1 0 0}$ & $\mathbf{1 2 0 0}$ & $\mathbf{1 3 0 0}$ \\
\hline B & Pressure & Bar & $\mathbf{0 . 3}$ & $\mathbf{0 . 4}$ & $\mathbf{0 . 5}$ \\
\hline C & Speed & $\begin{array}{c}\text { Mm/m } \\
\text { in }\end{array}$ & 300 & 400 & 500 \\
\hline
\end{tabular}

Table 2: control factor \& theirs level used in experiment

\subsection{Surface Roughness Measurement :}

The Surface Roughness for all trial runs is measured with Profilometer named as sj-201P. After the measuring number of samples through Surface Roughness tester (SJ -201P) In which we have measured L1, L2, L3 sample lengths and considered their average value in terms of length in $\mathrm{mm}$. in figure we have described measuring Surface Roughness of the sample and in table shown results of Surface Roughness test according to experiment.

\subsection{Specification of Mitutoyo surface tester SJ-201P[5] :}

\begin{tabular}{|ll|}
\hline & Measurement range $: 350 \mu \mathrm{m}$ \\
$>$ & Tip radius $: 5 \mu \mathrm{m}$ \\
$>$ & Measuring force $: 4 \mathrm{mN}($ Mega Newton) \\
$>$ & Radius of skid curvature $: 40 \mathrm{~mm}$ \\
$>$ & Tratector drive range: $21 \mathrm{~mm}$ \\
& to $0.5 \mathrm{~mm} / \mathrm{s}$ \\
& $\quad$ AC Peturn: $0.8 \mathrm{~mm} / \mathrm{s}$ \\
$>$ &
\end{tabular}

Table 3: Specification of surface tester SJ-201P

\begin{tabular}{|c|c|c|c|c|}
\hline $\begin{array}{c}\text { Trail } \\
\text { No }\end{array}$ & $\mathbf{A}$ & B & $\mathbf{C}$ & D \\
\hline 1 & 1 & 1 & 1 & 1 \\
\hline 2 & 1 & 1 & 2 & 2 \\
\hline 3 & 1 & 1 & 3 & 3 \\
\hline 4 & 1 & 2 & 1 & 2 \\
\hline 5 & 1 & 2 & 2 & 3 \\
\hline 6 & 1 & 2 & 3 & 1 \\
\hline 7 & 1 & 3 & 1 & 3 \\
\hline 8 & 1 & 3 & 2 & 1 \\
\hline 9 & 1 & 3 & 3 & 2 \\
\hline 10 & 2 & 1 & 1 & 2 \\
\hline 11 & 2 & 1 & 2 & 3 \\
\hline 12 & 2 & 1 & 3 & 1 \\
\hline 13 & 2 & 2 & 1 & 3 \\
\hline 14 & 2 & 2 & 2 & 1 \\
\hline 15 & 2 & 2 & 3 & 2 \\
\hline 16 & 2 & 3 & 1 & 1 \\
\hline 17 & 2 & 3 & 2 & 2 \\
\hline 18 & 2 & 3 & 3 & 3 \\
\hline 19 & 3 & 1 & 1 & 3 \\
\hline 15 & 3 & 1 & 2 & 1 \\
\hline 21 & 3 & 1 & 3 & 2 \\
\hline 22 & 3 & 2 & 1 & 1 \\
\hline 23 & 3 & 2 & 2 & 2 \\
\hline 24 & 3 & 2 & 3 & 3 \\
\hline 20 & 3 & 3 & 1 & 2 \\
\hline 26 & 3 & 3 & 2 & 3 \\
\hline 27 & 3 & 3 & 3 & 1 \\
\hline
\end{tabular}

Table 4: Lay out using L27 


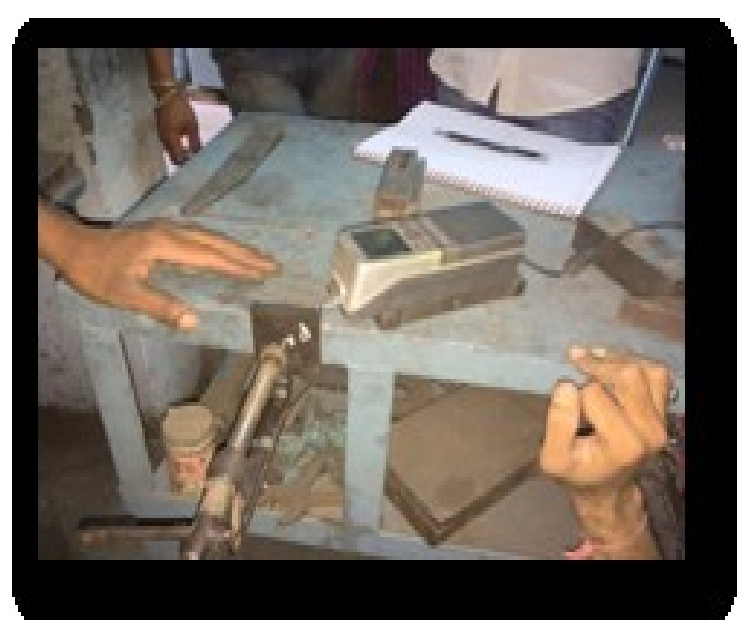

Figure 2: Surface Roughness Testing

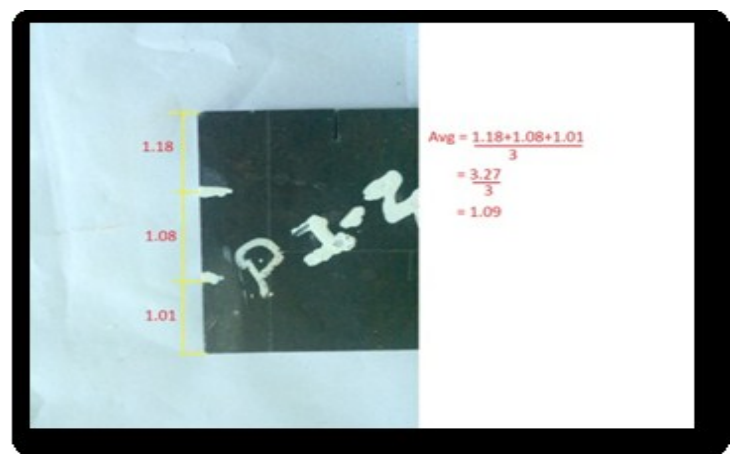

Figure 3: Avg of Surface Roughness of plate 1 and sub part 2

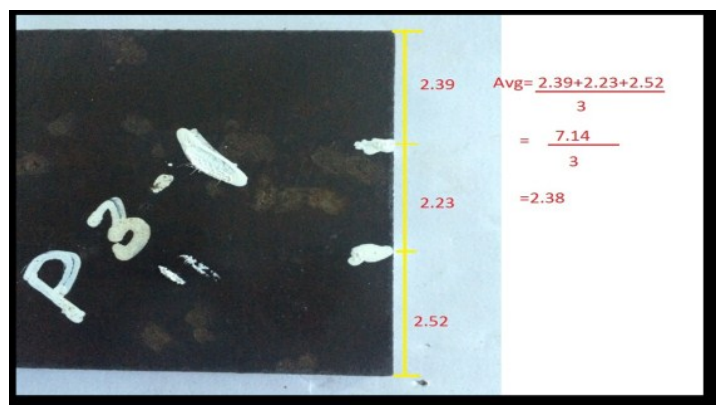

Figure 4: Avg of Surface Roughness of plate 3 and sub part 1

Table 5: Results of Surface Roughness obtained by experiment

\begin{tabular}{|c|c|c|c|c|c|}
\hline $\begin{array}{l}\text { Trail } \\
\text { No }\end{array}$ & Power(Watt) & $\begin{array}{c}\text { Pressure } \\
\text { (bar) }\end{array}$ & $\begin{array}{c}\text { Cutting } \\
\text { Speed } \\
(\mathrm{mm} / \mathrm{min})\end{array}$ & $\begin{array}{c}\text { Pulse } \\
\text { frequency } \\
(\mathrm{Hz})\end{array}$ & $\begin{array}{c}\text { Roughness } \\
\text { (micron) }\end{array}$ \\
\hline 1 & 1100 & 0.3 & 300 & 15 & 2.03 \\
\hline 2 & 1100 & 0.3 & 400 & 20 & 1.09 \\
\hline 3 & 1100 & 0.3 & 500 & 25 & 1.96 \\
\hline 4 & 1100 & 0.4 & 300 & 20 & 2.73 \\
\hline 5 & 1100 & 0.4 & 400 & 25 & 1.75 \\
\hline 6 & 1100 & 0.4 & 500 & 15 & 2.88 \\
\hline 7 & 1100 & 0.5 & 300 & 25 & 3.38 \\
\hline 8 & 1100 & 0.5 & 400 & 15 & 2.96 \\
\hline 9 & 1100 & 0.5 & 500 & 20 & 3.69 \\
\hline 10 & 1200 & 0.3 & 300 & 20 & 1.82 \\
\hline 11 & 1200 & 0.3 & 400 & 25 & 2.51 \\
\hline 12 & 1200 & 0.3 & 500 & 15 & 2.68 \\
\hline 13 & 1200 & 0.4 & 300 & 25 & 2.59 \\
\hline 14 & 1200 & 0.4 & 400 & 15 & 2.72 \\
\hline 15 & 1200 & 0.4 & 500 & 20 & 3.04 \\
\hline 16 & 1200 & 0.5 & 300 & 15 & 2.48 \\
\hline 17 & 1200 & 0.5 & 400 & 20 & 3.08 \\
\hline 18 & 1200 & 0.5 & 500 & 25 & 3.46 \\
\hline 19 & 1300 & 0.3 & 300 & 25 & 2.38 \\
\hline 20 & 1300 & 0.3 & 400 & 15 & 2.14 \\
\hline 21 & 1300 & 0.3 & 500 & 20 & 1.93 \\
\hline 22 & 1300 & 0.4 & 300 & 15 & 2.47 \\
\hline 23 & 1300 & 0.4 & 400 & 20 & 2.57 \\
\hline 24 & 1300 & 0.4 & 500 & 25 & 3.25 \\
\hline 25 & 1300 & 0.5 & 300 & 20 & 2.65 \\
\hline 26 & 1300 & 0.5 & 400 & 25 & 3.98 \\
\hline 27 & 1300 & 0.5 & 500 & 15 & 3.59 \\
\hline
\end{tabular}




\subsection{Kerf Width Measurement :}

The Kerf Width measurement was done by equipment using by digital camera and image tool programs. In which first digital camera was used to take the photographs of top bottom cut kerf of test piece, after that the photographs were transmitted in computer then after uploaded in image tool software for used to measure length along kerf gap[6].

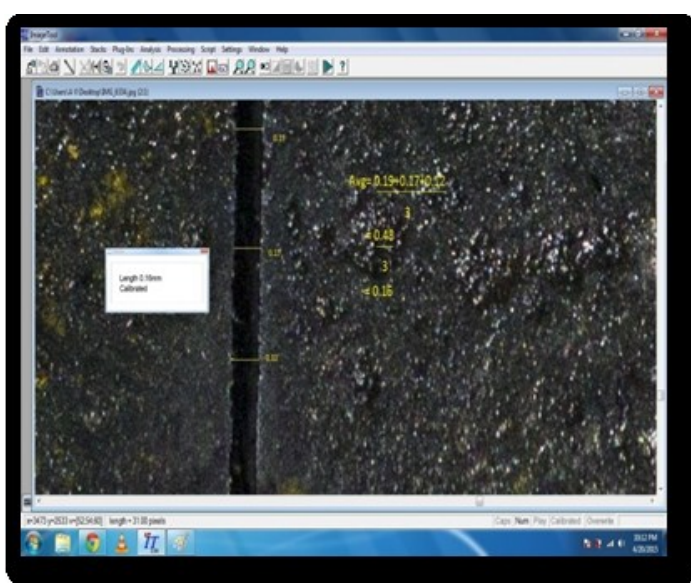

Figure 5: Kerf Width Analysis for plate no1 cut no7 by Image tool 3.0

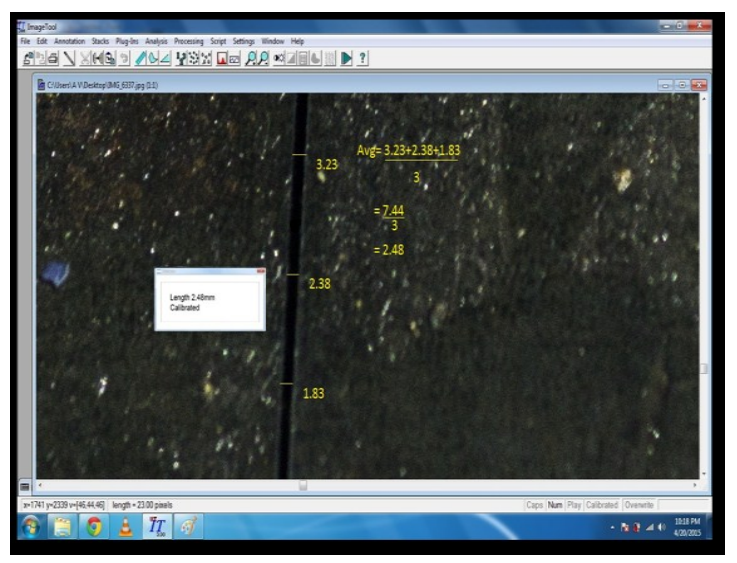

Figure 6: Kerf Width Analysis for plate no3 cut no9 by Image tool 3.0
Table 6: Results of Kerf Width obtained by experiment

\begin{tabular}{|c|c|c|c|c|c|}
\hline $\begin{array}{l}\text { Trail } \\
\text { No }\end{array}$ & Power(Watt) & $\begin{array}{l}\text { Pressure } \\
\text { (bar) }\end{array}$ & $\begin{array}{c}\text { Cutting } \\
\text { Speed } \\
(\mathrm{mm} / \mathrm{min})\end{array}$ & $\begin{array}{c}\text { Pulse } \\
\text { frequency } \\
(\mathbf{H z})\end{array}$ & $\begin{array}{l}\text { Kerfs } \\
\text { Width } \\
(\mathrm{mm})\end{array}$ \\
\hline 1 & 1100 & 0.3 & 300 & 15 & 0.40 \\
\hline 2 & 1100 & 0.3 & 400 & 20 & 0.22 \\
\hline 3 & 1100 & 0.3 & 500 & 25 & 0.28 \\
\hline 4 & 1100 & 0.4 & 300 & 20 & 0.19 \\
\hline 5 & 1100 & 0.4 & 400 & 25 & 0.87 \\
\hline 6 & 1100 & 0.4 & 500 & 15 & 0.33 \\
\hline 7 & 1100 & 0.5 & 300 & 25 & 0.16 \\
\hline 8 & 1100 & 0.5 & 400 & 15 & 1.89 \\
\hline 9 & 1100 & 0.5 & 500 & 20 & 0.37 \\
\hline 10 & 1200 & 0.3 & 300 & 20 & 0.82 \\
\hline 11 & 1200 & 0.3 & 400 & 25 & 0.56 \\
\hline 12 & 1200 & 0.3 & 500 & 15 & 0.93 \\
\hline 13 & 1200 & 0.4 & 300 & 25 & 0.54 \\
\hline 14 & 1200 & 0.4 & 400 & 15 & 0.78 \\
\hline 15 & 1200 & 0.4 & 500 & 20 & 0.48 \\
\hline 16 & 1200 & 0.5 & 300 & 15 & 0.90 \\
\hline 17 & 1200 & 0.5 & 400 & 20 & 1.29 \\
\hline 18 & 1200 & 0.5 & 500 & 25 & 0.37 \\
\hline 19 & 1300 & 0.3 & 300 & 25 & 1.13 \\
\hline 20 & 1300 & 0.3 & 400 & 15 & 0.51 \\
\hline 21 & 1300 & 0.3 & 500 & 20 & 1.86 \\
\hline 22 & 1300 & 0.4 & 300 & 15 & 1.29 \\
\hline 23 & 1300 & 0.4 & 400 & 20 & 0.49 \\
\hline 24 & 1300 & 0.4 & 500 & 25 & 2.28 \\
\hline 25 & 1300 & 0.5 & 300 & 20 & 1.47 \\
\hline 26 & 1300 & 0.5 & 400 & 25 & 1.26 \\
\hline 27 & 1300 & 0.5 & 500 & 15 & 2.48 \\
\hline
\end{tabular}




\section{DISCUSSION AND CONCLUSION :}

\subsection{Discussion about Surface Roughness:}

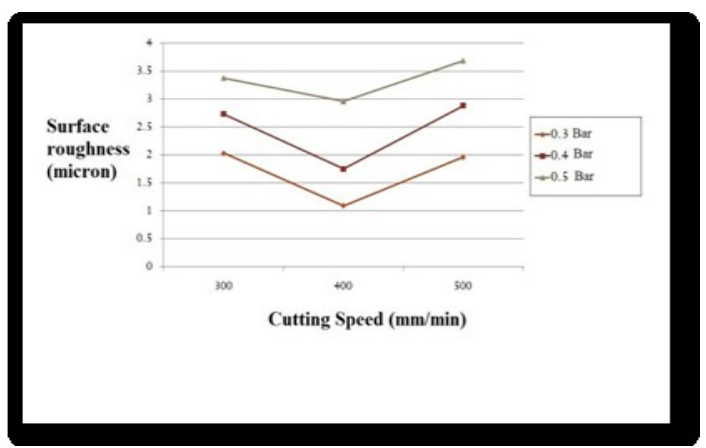

Figure 7: Graph of Cutting speed Vs Roughness with Laser Power1100watt

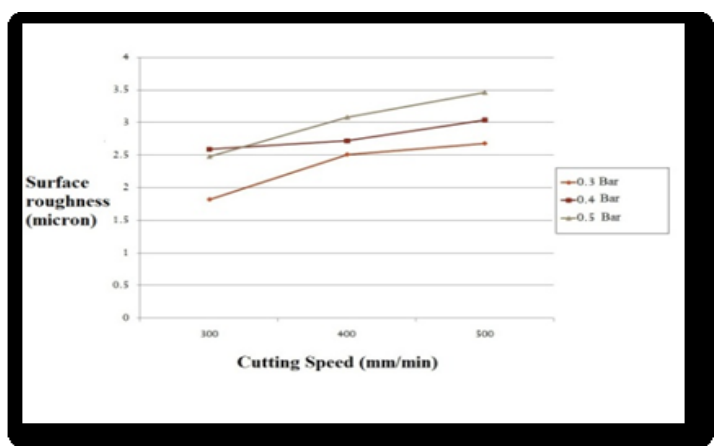

Figure 8: Graph of Cutting speed Vs Roughness with Laser Power1200watt

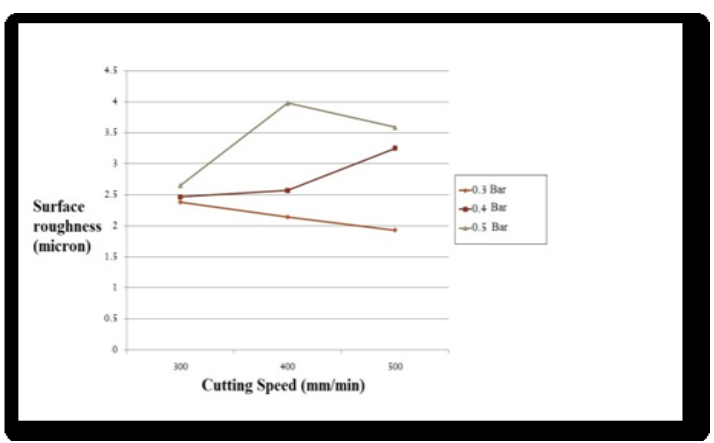

Figure 9: Graph of Cutting speed Vs Roughness with Laser Power1300watt

\subsection{Conclusion of Surface Roughness:}

After studied the performance the of $\mathrm{CO} 2$ Laser Cutting of weldox- 700 for $6 \mathrm{~mm}$ thickness with oxygen assistant gas cut by $\mathrm{CO} 2$ Laser Cutting machine made by Trumpf Laser Technology. After the experiment the cut quality was defined by focusing on Surface Roughness.
When the Laser Power is minimum like 1100watt, Cutting speed is medium and the gas pressure is medium we get best Surface Roughness like 1.09micron.

But when the Laser Power and Cutting speed are increased we get rough Surface Roughness and we seen it in 1200watt Cutting speed and 1300watt Laser Power graphs, and when the Laser Power is maximum like 1300watt get maximum Surface Roughness up to $\mathbf{3 . 9 8 m i c r o n . ~}$

\begin{tabular}{|c|c|c|c|c|c|}
\hline $\begin{array}{c}\text { Sr. } \\
\text { No }\end{array}$ & $\begin{array}{c}\text { Laser } \\
\text { Power } \\
\text { (watt) }\end{array}$ & $\begin{array}{c}\text { Pressure } \\
\text { (bar) }\end{array}$ & $\begin{array}{c}\text { Cutting } \\
\text { Speed } \\
(\mathrm{mm} / \mathrm{min})\end{array}$ & $\begin{array}{c}\text { Pulse } \\
\text { frequency } \\
\text { (Hz) }\end{array}$ & $\begin{array}{c}\text { Surface } \\
\text { Roughness } \\
\text { (micron) }\end{array}$ \\
\hline 1 & 1100 & 0.3 & 400 & 20 & 1.09 \\
\hline 2 & 1300 & 0.5 & 400 & 25 & 3.98 \\
\hline
\end{tabular}

\subsection{Discussion about Kerf Width:}

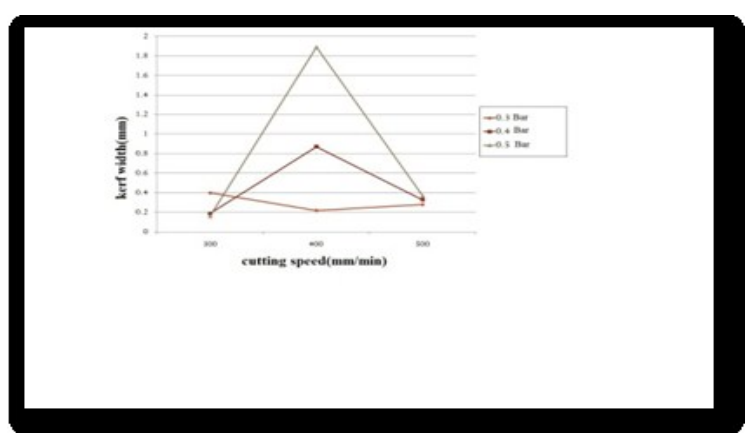

Figure 10: Graph of Cutting speed Vs Kerf Width with Laser Power1100watt

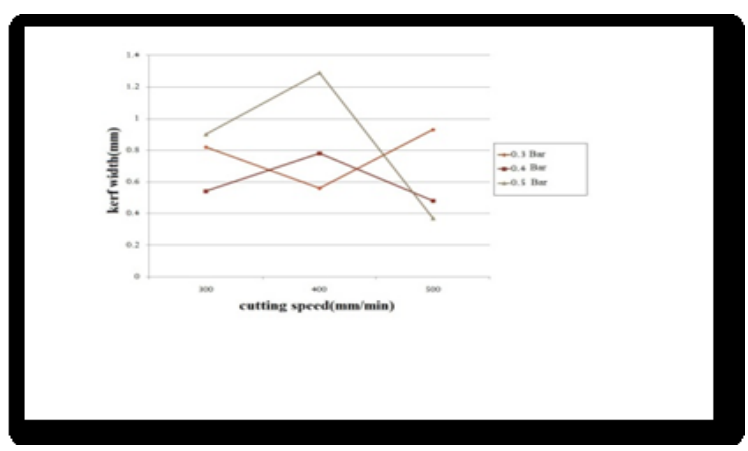

Figure 11: Graph of Cutting speed Vs Kerf Width with Laser Power1200watt 


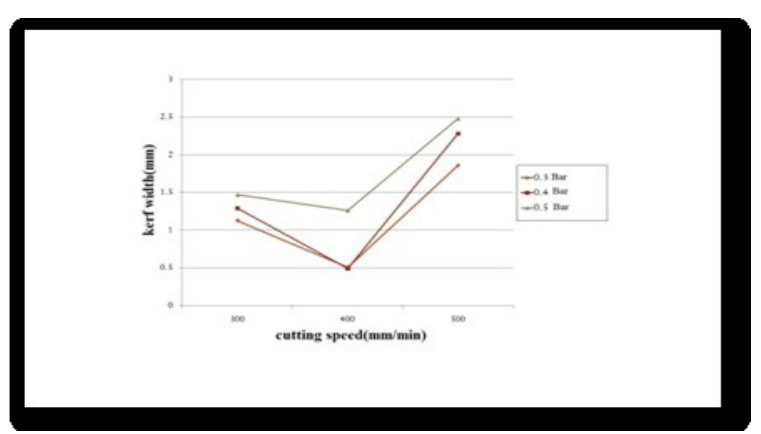

Figure 12: Graph of Cutting speed Vs Kerf Width with Laser Power1300watt

\subsection{Conclusion of Kerf Width :}

After studied the performance the of $\mathrm{CO} 2$ Laser Cutting of weldox- 700 for $6 \mathrm{~mm}$ thickness with oxygen assistant gas cut by $\mathrm{CO} 2$ Laser Cutting machine made by Trumpf Laser Technology. After the experiment the cut quality was defined by focusing on Surface Roughness

In which when the Laser Power is Minimum like 1100watt and the Cutting speed is minimum 300 and gas pressure is medium 0.5 we get Minimum Kerf Width like $\mathbf{0 . 1 6} \mathbf{m m}$.

When the speed and Power are increased the Kerf Width is increased. When the Power is highest 1300watt and Cutting speed is highest 500 Kerf Widths is increased up to $\mathbf{2 . 4 8} \mathbf{m m}$.

[7] Gould, R. Gordon (1959). "The LASER, Light Amplification by Stimulated Emission of Radiation". In Franken, P.A. and Sands, R.H. (Eds.). The Ann Arbor Conference on Optical Pumping, the University of Michigan, 15 June through 18 June 1959. p. 128.OCLC 02460155 .

[8] Chu, Steven; Townes, Charles (2003). "Arthur Schawlow". In Edward P. Lazar (ed.),.Biographical Memoirs. vol. 83. National Academy of Sciences. p. 202. ISBN 0-309-08699-X

[9] Nolen,jim;Derek verno."The carbon Dioxide Laser". Davidson Physics. Retried17 Aug 2014

[10] https://www.idc online.com/technical_references/pdfs/mechanical_engine ering/Parametric\%20investigation

[11] Achievements in Materials and manufacturing Engineering 54/1 (2012) 128-134

[12] Jia Deli, You Bo (2010) - "An intelligent control strategy for plasma arc Cutting technology" Harbin University of Science and Technology, Harbin 150080, China

[13] Jiayou Wang \&Zhengyu Zhu \&Conghui He \&Feng Yang (2010) - "Effect of dual swirling plasma arc Cutting parameters on kerf characteristics" Int J Mater Form (2011) 4:39-43

[14] http://www.revtn.ro/pdf4-Radovanovic

[15] https://www.idc online.com/technical_references/pdfs/mechanical_engine ering/Parametric\%20investigation.pdf

[16] http://www.tribology.fink.rs/journals/2012/2012-4/7.pdf

[17] http://www.ijsea.com/archive/volume2/issue6/IJSEA020 61003.html

[18] http://www.ijetae.com/files/Volume3Issue4/IJETAE_041 3_35.pdf

[19] K.A. Ghany, M. Newishy, Cutting of $1.2 \mathrm{~mm}$ thick austenitic stainless steel sheet using pulsed and $\mathrm{CW} \mathrm{Nd}$ YAG Laser, Journal of Material Processing Technology 168 (2005) 438-447

\begin{tabular}{|c|c|c|c|c|c|}
\hline $\begin{array}{c}\text { Sr. } \\
\text { No }\end{array}$ & $\begin{array}{c}\text { Laser } \\
\text { Power } \\
\text { (watt) }\end{array}$ & $\begin{array}{c}\text { Pressure } \\
\text { (bar) }\end{array}$ & $\begin{array}{c}\text { Cutting } \\
\text { Speed } \\
(\mathbf{m m} / \mathbf{m i n})\end{array}$ & $\begin{array}{c}\text { Pulse } \\
\text { frequency } \\
(\mathbf{H z})\end{array}$ & $\begin{array}{c}\text { Kerf } \\
\text { Width } \\
(\mathbf{m m})\end{array}$ \\
\hline 1 & 1100 & 0.5 & 300 & 25 & 0.16 \\
\hline 2 & 1300 & 0.5 & 500 & 15 & 2.48 \\
\hline
\end{tabular}

[20] S.-L. Chen, The effects of high-pressure assistant-gas flow on high Power CO2 Laser Cutting, Journal of Material Processing Technology 88 (1999) 57-66

21] N. Raja ram, J.S. Ahmad, S.H. Cheraghi, -CO2 Laser cut quality of 4130 steell, International Journal of Machine Tools and Manufacture 43 (2003) 351-358.

[22] F. Caiazzo, F. Curcioa, G. Daurelio, F. Memola Capece Minutolo, - Laser Cutting of different polymeric plastics

[23] M. Zaied, E. Bayraktar, D. Katundi, M. Boujelbene, I. Miraoui, - Effect of Laser Cutting parameters on surface quality of low carbon steel (S235)\|, Journal of

\section{References:}

[1] http://en.wikipedia.org/wiki/Laser

[2] George Chryssolouris, "Laser Machining", New, Springer-Verlag, 1991;

[3] www.weldox.com

[4] http://support.minitab.com/en-us/minitab/17/topiclibrary/modeling-statistics/doe/taguchi-designs/taguchidesigns/

[5] https://www.atecorp.com/ATECorp/media/pdfs/datasheets/Mitutoyo-SJ-201P_Datasheet.pdf

[6] http://compdent.uthscsa.edu/dig/itdesc.html
[24] N. Rajendran, M.B. Pate, The effect of Laser beam velocity on cut quality and surface temperature, American Society of Mechanical Engineers, Heat Transfer Division 104 (1988) 121-127

[25] Schuocker, D. (1998). Handbook of the Euro Laser Academy.

[26] S. Crouch and Thomas Savery, The Miner's Friend: Or an Engine to Raise Water by Fire, 1827, Page 12-16 
[27] Phillip J. Ross, Taguchi Techniques For Quality Engineering, Tata McGraw Hill, $2^{\text {nd }}$ Edition,2012 Page 91-179

[28] Avanish Kumar Dubey, and Vinod Yadava. Optimization of kerf quality during pulsed Laser Cutting of aluminium alloy sheet. Journal of materials processing technology, pages 412-418, 2008.

[29] A.Riveiro et al. The Role of the Assist Gas Nature in Laser Cutting of Aluminum Alloys. Physics Procedia, pages 548-554, 2011.

[30] Arindam Ghosal, and Alakesh Manna. Response surface method based optimization of ytterbium fiber Laser parameter during machining of Al/AL2O3-MMC. Opticsand Laser Technology, pages 67-76, 2013.

[31] http://www.annualreviews.org/doi/abs/10.1146/annurev. pc.34.100183.003013

[32] http://www.rp-photonics.com/solid_state_Lasers.html

[33] http://www.rp-photonics.com/fiber_Lasers.html

[34] http://www.rpphotonics.com/semiconductor_Lasers.html

[35] http://www.colby.edu/chemistry/PChem/lab/DyeLaser.p df

[36] http://www.rp-photonics.com/free_electron_Lasers.html

[37] http://www2.isye.gatech.edu/ roshan/publications/Taguc hi.pdf

[38] http://www2.kuas.edu.tw/prof/shihtao/qms/Powerpoint/f oster\%20text/Ch07S-new.ppt 